Prepared for the U.S. Department of Energy

under Contract DE-AC05-76RL01830

\title{
Automated Thermal Image Processing for Detection and Classification of Birds and Bats
}

\section{FY2012 Annual Report}

Offshore Wind Technology Assessment
CA Duberstein
DJ Virden
S Matzner
J Myers
VI Cullinan
AR Maxwell

September 2012

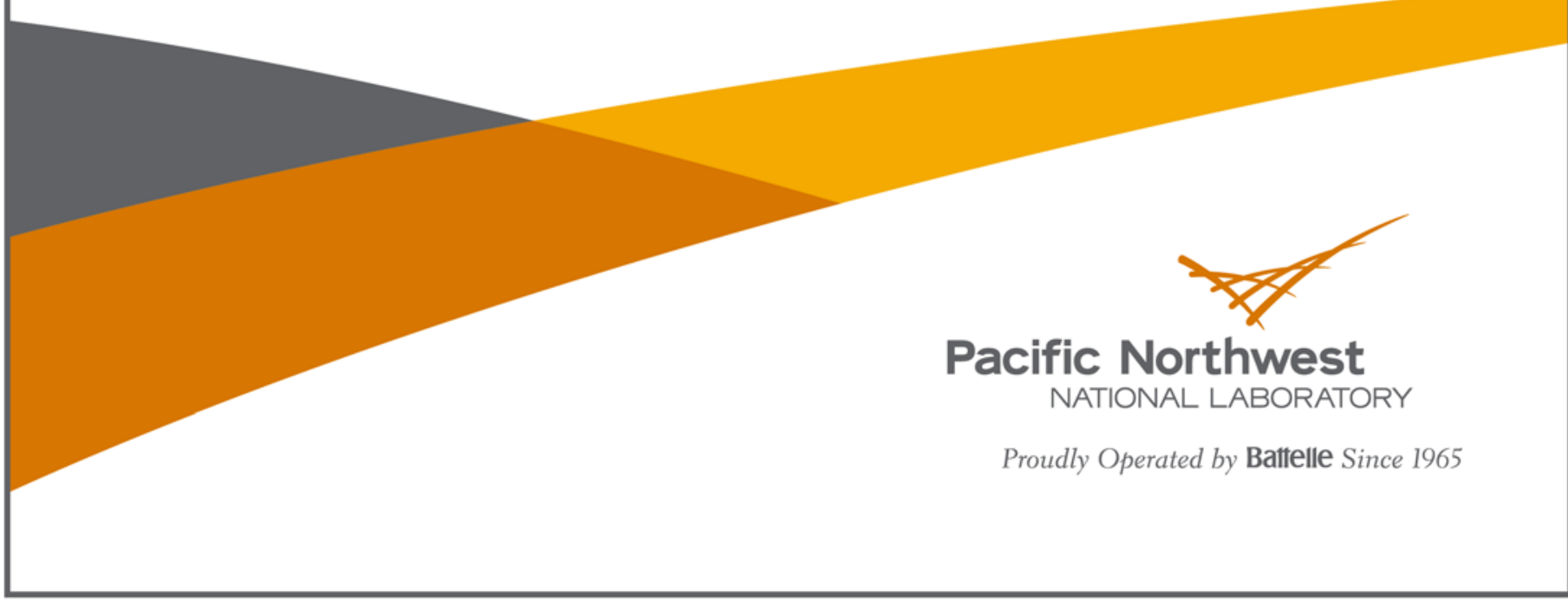




\title{
DISCLAIMER
}

This report was prepared as an account of work sponsored by an agency of the United States Government. Neither the United States Government nor any agency thereof, nor Battelle Memorial Institute, nor any of their employees, makes any warranty, express or implied, or assumes any legal liability or responsibility for the accuracy, completeness, or usefulness of any information, apparatus, product, or process disclosed, or represents that its use would not infringe privately owned rights. Reference herein to any specific commercial product, process, or service by trade name, trademark, manufacturer, or otherwise does not necessarily constitute or imply its endorsement, recommendation, or favoring by the United States Government or any agency thereof, or Battelle Memorial Institute. The views and opinions of authors expressed herein do not necessarily state or reflect those of the United States Government or any agency thereof.

\author{
PACIFIC NORTHWEST NATIONAL LABORATORY \\ operated by \\ BATTELLE \\ for the \\ UNITED STATES DEPARTMENT OF ENERGY \\ under Contract DE-AC05-76RL01830
}

Printed in the United States of America

$$
\begin{aligned}
& \text { Available to DOE and DOE contractors from the } \\
& \text { Office of Scientific and Technical Information, } \\
& \text { P.O. Box } 62 \text {, Oak Ridge, TN } 37831-0062 \text {; } \\
& \text { ph: }(865) 576-8401 \\
& \text { fax: (865) 576-5728 } \\
& \text { email: reports@adonis.osti.gov }
\end{aligned}
$$

Available to the public from the National Technical Information Service

5301 Shawnee Rd., Alexandria, VA 22312 ph: (800) 553-NTIS (6847)

email: orders@ntis.gov $<$ http://www.ntis.gov/about/form.aspx $>$ Online ordering: http://www.ntis.gov 


\section{Automated Thermal Image Processing for Detection and Classification of Birds and Bats}

\section{FY2012 Annual Report}

\section{Offshore Wind Technology Assessment}

$\begin{array}{ll}\text { CA Duberstein } & \text { DJ Virden } \\ \text { S Matzner } & \text { J Meyer } \\ \text { VI Cullinan } & \text { AR Maxwell }\end{array}$

September 2012

Prepared for the U.S. Department of Energy under Contract DE-AC05-76RL01830 


\section{Preface}

During FY2012, internal funding from the Pacific Northwest National Laboratory's Lab Directed Research and Development (LDRD) Program provided support to begin exploring the development of software that could identify objects of interest (birds and bats) sensed with thermal imaging video equipment. Previous research indicated that the use of infrared video cameras was an effective method to survey the sky for birds and bats. Unlike traditional methods that require a human observer recording events as they are observed, video recording provides a real-time archive of what was observed and could be conducted at remote locations. However, the identification of observed phenomena still requires a trained observer viewing the video, which becomes both time consuming and expensive, and, like traditional methods, is still prone to observer bias. The research team began by acquiring existing thermal video files gathered by Sid Gauthreax for a previous research project at the Clemson University Radar Ornithology Laboratory. A new algorithm for automatically detecting birds and bats in infrared video was designed and implemented within MATLAB. The algorithm design is a unique combination of video peak store (VPS) processing, region growing, and perceptual grouping techniques. Birds, bats, and other warm targets moving through the camera's field of view (FOV) produce bright spots in the video that change position from frame to frame. VPS is the process of storing the peak intensity of each pixel in the video over the course of a fixed time window into a single image. The resulting image then contains the history of a target's motion, or its track, through the camera's FOV. VPS is usually done with a dedicated hardware device, but we wrote our own code within MATLAB to do the VPS processing.

The algorithm then detects tracks in the set of VPS images produced from a video recording. Conceptually, a track is composed of a series of objects. An object is a spatially connected group of pixels that had peak values in the same frame. Individual pixels are first grouped into objects using a form of region growing that was tailored to this application. Objects then are combined into tracks using perceptual grouping, a general method of image processing inspired by human visual perception. In our algorithm, similar objects that lie in a line or along a curve are grouped together as a track. Much of the algorithm development involved defining the terms similar and lies in a line or along a curve in an appropriate mathematical form. Each track is identified by the time it starts in the recorded video for independent verification with observer annotations. The text file also contains a number of measures of each track, such as the mean size and intensity of the objects in the track, and the sinuosity of the track. The sinuosity is a measure of the change in direction between successive objects in a track.

Although efforts within this project showed that processing of video files to extract information could be automated, further work including efficiency testing and development of track classification methods were logical next steps addressed with funding from the U.S. Department of Energy Office of Energy Efficiency and Renewable Energy. 


\section{Summary}

Surveying wildlife at risk from offshore wind energy development is difficult and expensive. Infrared video can be used to record birds and bats that pass through the camera view, but it is also time consuming and expensive to review video and determine what was recorded. We proposed to conduct algorithm and software development to identify and to differentiate thermally detected targets of interest that would allow automated processing of thermal image data to enumerate birds, bats, and insects. During FY2012 we developed computer code within MATLAB to identify objects recorded in video and extract attribute information that describes the objects recorded. We tested the efficiency of track identification using observer-based counts of tracks within segments of sample video. We examined object attributes, modeled the effects of random variability on attributes, and produced data smoothing techniques to limit random variation within attribute data. We also began drafting and testing methodology to identify objects recorded on video.

We also recorded approximately 10 hours of infrared video of various marine birds, passerine birds, and bats near the Pacific Northwest National Laboratory (PNNL) Marine Sciences Laboratory (MSL) at Sequim, Washington. A total of 6 hours of bird video was captured overlooking Sequim Bay over a series of weeks. An additional 2 hours of video of birds was also captured during two weeks overlooking Dungeness Bay within the Strait of Juan de Fuca. Bats and passerine birds (swallows) were also recorded at dusk on the MSL campus during nine evenings. An observer noted the identity of objects viewed through the camera concurrently with recording. These video files will provide the information necessary to produce and test software developed during FY2013. The annotation will also form the basis for creation of a method to reliably identify recorded objects. 


\section{Acknowledgments}

The Wind and Water Power Program within the U.S. Department of Energy-Office of Energy Efficiency and Renewable Energy funded this research. 


\section{Acronyms and Abbreviations}

$\begin{array}{ll}\text { DOE } & \text { U.S. Department of Energy } \\ \text { EERE } & \text { Office of Energy Efficiency and Renewable Energy } \\ \text { FOV } & \text { field of view } \\ \text { GLM } & \text { general linear model } \\ \text { IQR } & \text { interquartile range } \\ \text { IR } & \text { infrared } \\ \text { LDRD } & \text { Laboratory Directed Research and Development } \\ \mu \mathrm{m} & \text { micron(s) } \\ \text { MSL } & \text { Marine Sciences Laboratory } \\ \text { PNNL } & \text { Pacific Northwest National Laboratory } \\ \text { VPS } & \text { video peak store }\end{array}$




\section{Contents}

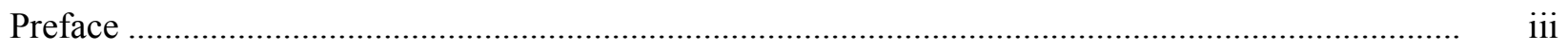

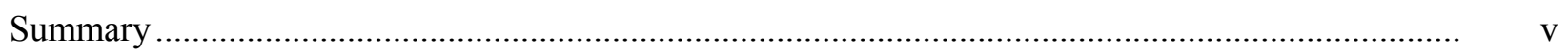

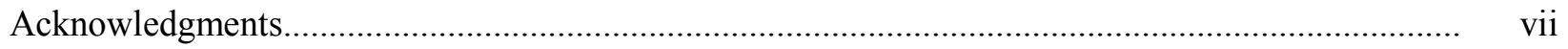

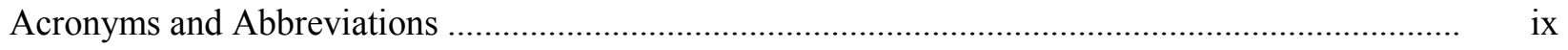

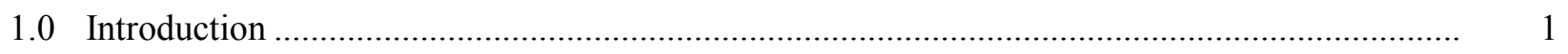

2.0 Annotated Video Capture ............................................................................................... 2

3.0 Algorithm Description, Testing, and Improvement .......................................................... 2

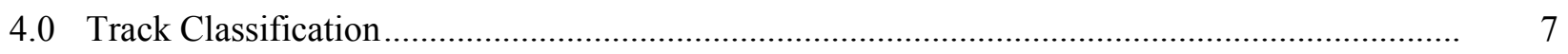

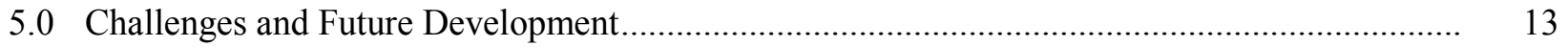

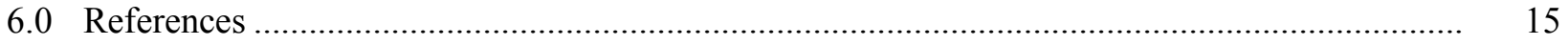




\section{Figures}

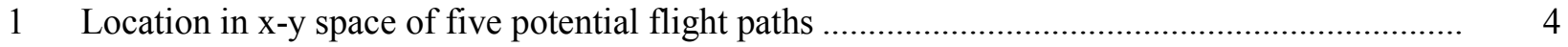

2 Location in $x-y$ space based on the quadratic flight path and the same $x-y$ location with

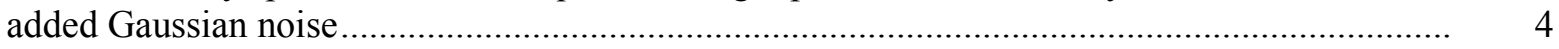

3 Boxplot of the IQR before smoothing for each model........................................................ 5

4 Interaction plots for the delta statistic for the IQR of the change in direction

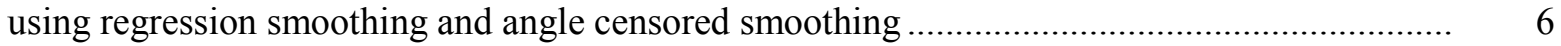

5 Interaction plots for the delta statistic for the IQR of the change in direction using moving

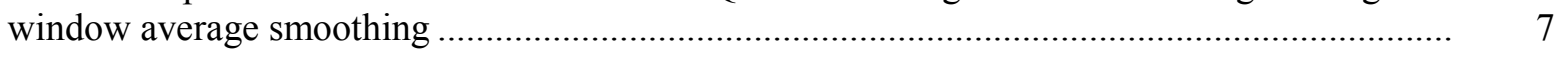

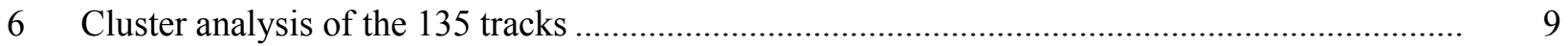

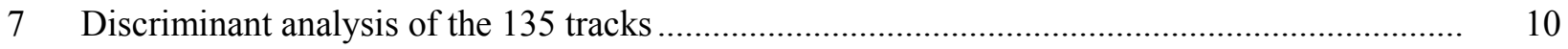

8 A new flight path used to challenge the discriminant function classification process................. 12

9 Location in canonical space of the unknown challenge track.................................................... 13

\section{Tables}

1 Descriptive statistics of IQR with and without random noise before smoothing....................... 5

2 Correlation matrix of the descriptive statistics of the change in direction using the moving

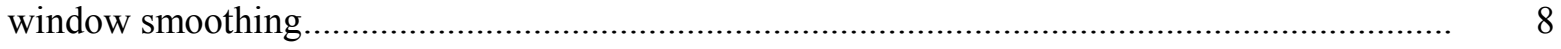

3 Similarity matrix between cluster centroids. ............................................................... 10

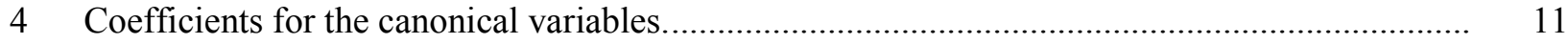

5 Mean and standard deviation for standardizing the descriptive statistics on the change in

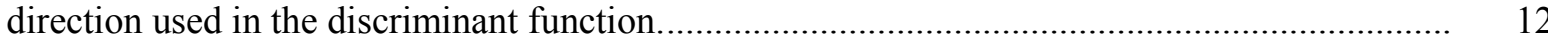

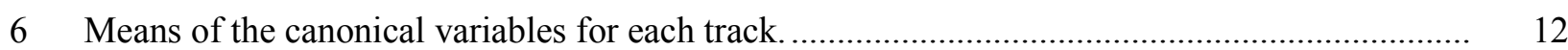

7 Distance from each centroid and probability of membership to each track category for the challenge track. 


\subsection{Introduction}

The primary method to assess environmental risk of a wind energy development to flying fauna (birds and bats) is to enumerate birds and bats that fly through the rotor-swept zone using marine radar (Davenport 2010; d'Entremont 2010; Johnson 2010; Svedlow 2011). Information currently provided by radars would include the number of targets passing through the radar beam in a set amount of time, distance a target is from the radar (elevation or range depending on configuration), and trajectory or travel path. However, radar data alone does not allow researchers to effectively differentiate between targets of interest (birds, bats) and other flying objects (insects). The detection of insects skews risk calculations resulting in erroneous risk modeling.

One method successfully used to identify birds, bats, and insects detected by radar is the simultaneous deployment of a thermal-imaging camera with radar. Zehnder et al. (2001) used a passive infrared (IR) video camera to study nocturnal migration of birds in Sweden. Gauthreaux and Livingston (2006) also used a passive IR camera system coupled with vertically pointed radar to assess nighttime migration. The camera provided the path a target traveled ( $\mathrm{X}$ and $\mathrm{Y}$ dimensions) while the radar provided altitude ( $\mathrm{Z}$ dimension). Airborne targets (bats, birds, and insects) were recorded with both the radar and thermal camera. Target differentiation was accomplished by traits exhibited by the target. Birds were identified

as bright (i.e., warm) targets that flew in a relatively straight track, and their wing beats were displayed as echo modulation in both the radar and thermal imaging data. Insects also traveled in a straight path, but targets appeared dull (cool) in the thermal image data and did not display any modulation from wing beats. Bats were also bright as expected. Zehnder et al. (2001) concluded bat and bird observations would be difficult to distinguish.

However, foraging bats have an erratic flight pattern and their irregular tracks were discernible on the thermal video (S. A. Gauthreaux Jr., Clemson University, personal communication, February 2012). The occurrence of insects in radar survey data is still an ongoing issue that has not been resolved and is often not addressed by researchers. Both Zehnder et al. (2001) and Gauthreaux and Livingston (2006) processed thermal imaging data manually during their study, and thermal imaging cameras are still used to proof radar ornithological data sets. However, manual processing of thermal imagery is time consuming and cost prohibitive, and the need for a tool to allow automated processing of thermal imagery has been identified within the wind energy research community (S. A. Gauthreaux Jr., Clemson University, personal communication, February 2012).

We proposed to conduct algorithm and software development to identify and to differentiate thermally detected targets of interest that would allow automated processing of thermal image data to enumerate birds, bats, and insects. Development of the capability to design, modify, and deploy thermal image processing techniques and tools would provide the scientific community with a tool to proof large volumes of data critical to siting wind energy projects in a time- and cost- efficient manner that is both objective and scientifically defensible. To accomplish this, during FY 2012, we gathered an annotated digital thermal video library, conducted further development on the Laboratory Directed Research and Development (LDRD) project video-processing algorithm, and performed sensitivity modeling to begin to assess track classification metrics. 


\subsection{Annotated Video Capture}

During initial project planning stages, an annotated video data library depicting bats and various types of birds was identified as a critical component to develop software that would enable the identification of birds and bats recorded with a digital thermal video camera system. Although the original video data provided a basis from which to demonstrate a process the identification of target tracks, differences in study design and data capture protocol resulted in video files that did not provide information necessary to develop software capable of assessing risk to bird and bat populations from offshore developments. Therefore, we undertook a field data collection effort to obtain a series of video files of birds, bats, and other airborne phenomena including seabirds representative of what might be expected offshore.

Video was recorded at multiple locations at or near the Pacific Northwest National Laboratory (PNNL) Marine Sciences Laboratory (MSL) at Sequim, Washington. Our video recording protocol was designed to record bird species that occur in nearshore marine environments that would represent those bird types that could be expected in an offshore environment. Video was recorded by a two-person team consisting of an observer and a camera operator with an Axsys Technologies FieldPro 5x Thermal Imager. The camera has a 320-pixel horizontal array and a 240-pixel vertical array. It had a horizontal angular field of view of $5.5^{\circ}$ and a vertical angular field of view of $4.1^{\circ}$. The thermal sensitivity is $0.04^{\circ} \mathrm{C}$, and the spectral range is $3-5 \mu \mathrm{m}$. We recorded at 30 frames per second directly onto a laptop hard drive. The camera was pointed just above horizontal during video recording. Concurrent with the recording, a visual survey was conducted of the sample airspace. The observer was equipped with a pair of image-stabilized binoculars mounted on a table-top tripod. The observer used a Python-based computer program to record targets as they passed and also described the targets' paths into the audio recorder to assist in later identification of tracks.

A total of 6 hours of bird video was captured overlooking Sequim Bay over a series of weeks. An additional 2 hours of video of birds was also captured during two weeks overlooking Dungeness Bay within the Strait of Juan de Fuca. Airborne objects that produced tracks within video recorded during FY 2012 include birds, bats, insects, clouds, and airplanes. Additionally, waves, boats, and other moving phenomena were also recorded. Birds observed during video recording include gulls, terns, cormorants, waterfowl, shorebirds, and passerines. Bats and passerine birds (swallows) were also recorded at dusk on the MSL campus during nine evenings. The rate of observed targets varies widely from one bird or bat per 3 minutes of video to multiple dozens of gulls per minute.

\subsection{Algorithm Description, Testing, and Improvement}

The algorithm we created during the LDRD project provided proof-of-concept that target tracks could be identified and numerical data describing those tracks could be extracted from digital video. The algorithm functions by processing digital video frame by frame to identify pixel groups with similar thermal signature values. These pixels are then grouped into objects that are then associated with other objects near them in subsequent video frames within a predetermined time window to form tracks. Parameterization of the algorithm was based on the pre-existing sample video. Parameters, such as the amount of thermal signature similarity, proximity of objects in time and space within subsequent video frames, the definition of the "center of mass" of an object, and the length of time necessary to capture an 
entire object track, were defined by traits of the recorded objects. For example, the time window we selected to identify tracks was 10 seconds, as virtually all of the objects observed within Gauthreaux's sample video would pass entirely through the camera view in less than 10 seconds.

Following the LDRD effort, we tested the efficiency of track identification using observer-based counts of tracks within segments of sample video. Human observers viewing the video counted more tracks than were identified by the algorithm. This difference in count was in part a result of parameterization. The human brain can sense motion while viewing video and associate that motion into a target and track. Many tracks recorded by an observer included faint (less thermally intense) objects and objects visible for only a very short period of time. After defining a track using parameters of thermal intensity and number of frames an object has to be visible to form a track, the algorithm detected $100 \%$ of tracks without reporting any false positive tracks (creating tracks that did not appear to a human observer as a track). We also evaluated the classification of tracks based on the extracted attribute information. Original track attributes included measures of thermal intensity, object size, rate of travel across the view, and change in direction.

Birds and bats often have characteristic flight patterns. For instance, larger birds such as gulls typically fly in straighter lines than swallows and other small birds. Furthermore, bats display a very undulating flight. We theorized change of direction (i.e., track sinuosity) could be used as an attribute to classify tracks. Our measures of sinuosity are calculated by drawing a line connecting the centers of mass in subsequent images and measuring the angle created between lines in successive images. However, upon inspection of this method, it became apparent that measures of sinuosity produced by the algorithm were highly variable and did not appear to represent changes in flight path observed.

We hypothesized that the sinuosity values were being inflated from the calculation methods because, as a bird or bat flies through the camera view, wing flapping causes the pixel object to change shape. These apparent shape changes result in the geographic center of mass being calculated in slightly different locations within the object pixel group, resulting in large values of direction change for tracks that appear relatively straight. To determine the source of error, we simulated five tracks that represented typical flight paths of birds and bats that were observed within the sample video: linear, quadratic, sine, angle and turnaround (Figure 1). Gaussian noise was used to mimic the slight location change in X-Y space associated with calculating the center of mass of a pixilated object that changes shape slightly from frame to frame (Figure 2).

We determined that slight noise in the X-Y locations can cause greater than expected sinuosity for even relatively straight flight paths. The inadvertent increase in apparent direction change caused during data extraction can be removed by smoothing the data before calculating statistics. Three techniques were employed to smooth the noise added to the flight path: 1) regression of a moving window of $x-y$ locations, 2) moving window average of the change in direction, and 3) angle censoring defined as setting the value of direction change to zero when it was less than a specified value.

Descriptive statistics associated with the entire flight path were calculated and compared for the change in direction before and after smoothing using the moving window averaging and angle censoring techniques. For the regression smoothing technique, descriptive statistics were calculated and compared for the change in direction before smoothing and for the linear slope of the regression. Descriptive statistics calculated for each flight path included the minimum, maximum, mean, standard deviation, and quartiles (Q1, Q2, and Q3) of the data distribution; the interquartile range $(\mathrm{IQR}=\mathrm{Q} 3-\mathrm{Q} 1)$; the 
difference between the mean and median (central skewness); the range; and the sample distribution skewness and kurtosis. The number of times the sign changed between successive values of $\mathrm{C}_{t}$ before and after smoothing (ignoring zeros) and between successive values of the slope was also calculated. For the regression smoothing technique, the maximum absolute change between successive slopes divided by the absolute value of the median slope was calculated.

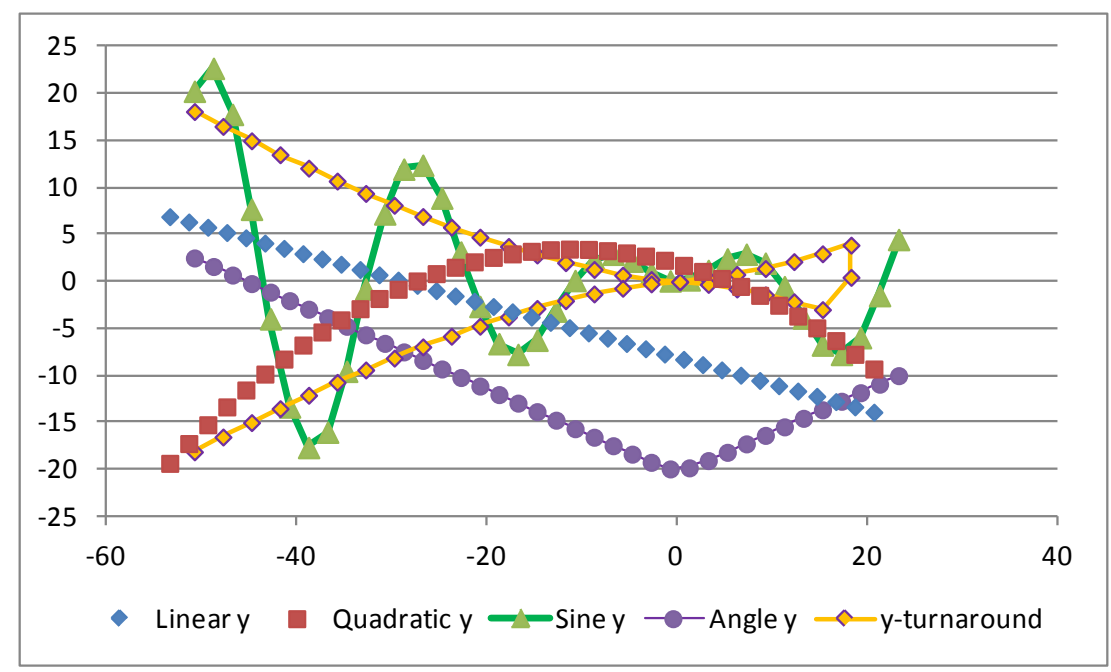

Figure 1. Location in $x-y$ space of five potential flight paths.

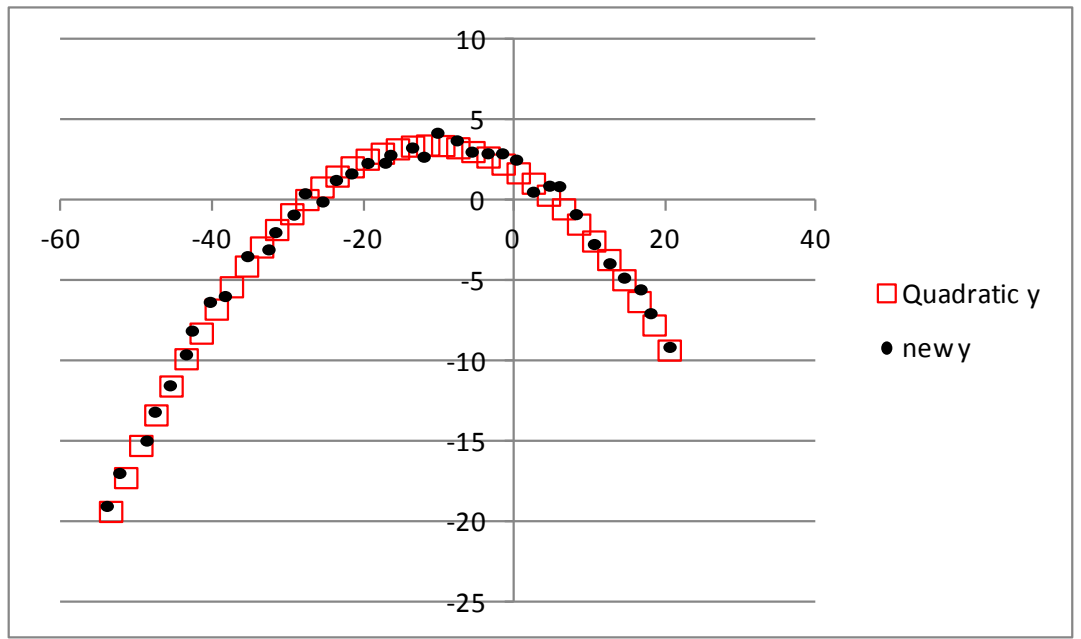

Figure 2. Location in $x-y$ space based on the quadratic flight path (open square) and the same $x-y$ location with added Gaussian noise (black dot).

Data for nine realizations of each flight path was generated. Before smoothing, the mean IQR of the five flight paths were all greater than the IQR of the sine wave without noise added (Table 1). The means were significantly different (analysis of variance [ANOVA], $p=0.004$ ) with the mean IQRs from the linear and angled models significantly greater than the mean IQR from the turnaround model. Random noise has the greatest effect on the IQR for those models with long stretches of straight flight paths (Figure 3). To compare smoothing techniques on the calculation of the IQR, the difference (delta) in the 
statistic before and after smoothing was calculated (i.e., original value - smoothed value). Large positive values of delta mean that the smoothed IQR has been pushed toward zero (successful smoothing). Small positive values of delta mean that the smoothing did not make much of a difference, and negative values of delta mean that the smoothing increased the IQR. Smoothing was conducted on three realizations of each of three different regression and moving average widths $(\mathrm{w}=4,5$, and $6 \mathrm{x}-\mathrm{y}$ values $)$ and three different angle censoring values $(\pi / 5, \pi / 6$, and $\pi / 8)$. Thus, a total of 81 simulations were conducted for each track.

Table 1. Descriptive statistics of IQR with and without random noise before smoothing.

\begin{tabular}{|l|c|c|c|c|c|c|}
\hline \multirow{2}{*}{ Model } & IQR & \multicolumn{5}{|c|}{ IQR Data Distribution with Random Noise } \\
\cline { 5 - 8 } & $\begin{array}{c}\text { Random } \\
\text { Noise }\end{array}$ & $\begin{array}{c}\text { Sample } \\
\text { Size }\end{array}$ & Mean & $\begin{array}{c}\text { Standard } \\
\text { Deviation }\end{array}$ & $\begin{array}{c}\text { Minimum } \\
\text { Value }\end{array}$ & $\begin{array}{c}\text { Maximum } \\
\text { Value }\end{array}$ \\
\hline Linear & 0.000 & 9 & 0.86 & 0.17 & 0.50 & 1.04 \\
\hline Angled & 0.001 & 9 & 0.81 & 0.14 & 0.68 & 1.03 \\
\hline Quadratic & 0.013 & 9 & 0.79 & 0.13 & 0.62 & 1.07 \\
\hline Turnaround & 0.021 & 9 & 0.62 & 0.10 & 0.47 & 0.76 \\
\hline Sine wave & 0.562 & 9 & 0.74 & 0.07 & 0.63 & 0.85 \\
\hline
\end{tabular}

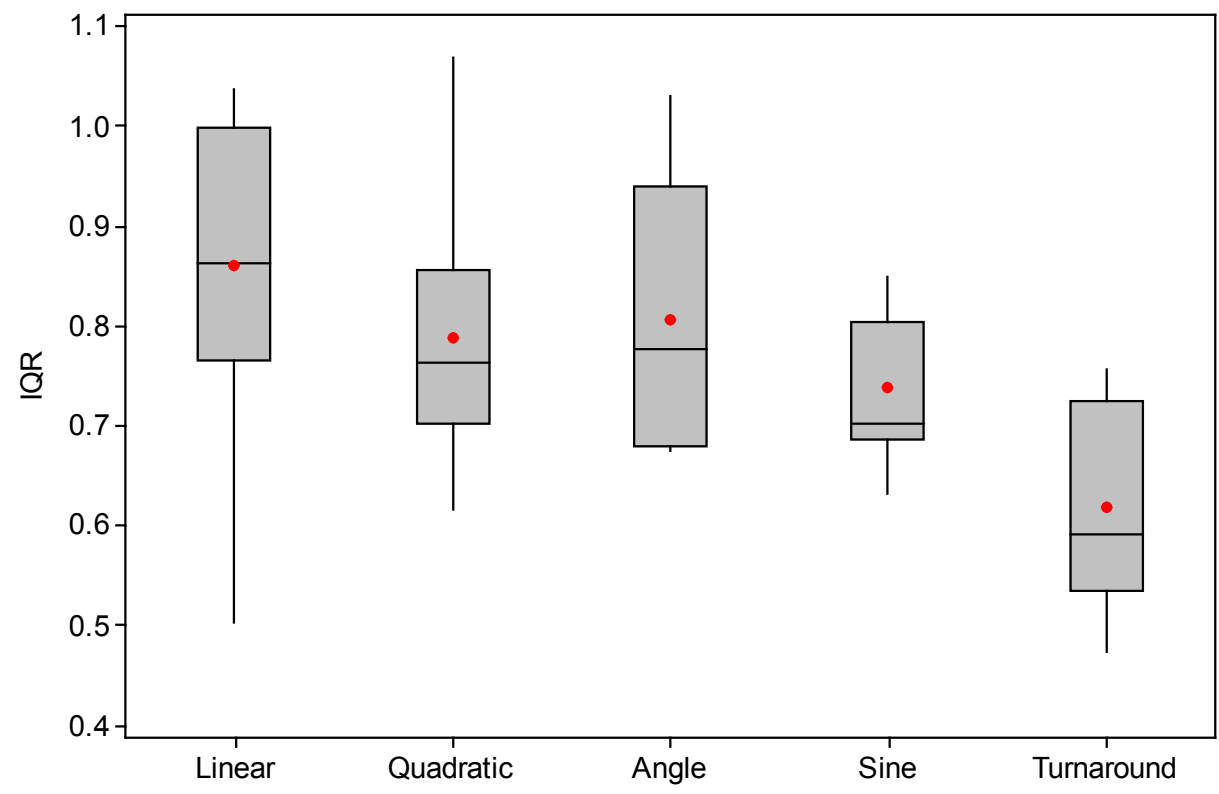

Figure 3. Boxplot of the IQR before smoothing for each model $(\mathrm{n}=9)$.

The intent of smoothing is to reduce the random noise without losing the basic characteristics of the flight path (track). A general linear model (GLM) of the main effects of track (i.e., linear, quadratic, and so on) and window size (or angle to censor) and their interaction was conducted using the delta statistics for each smoothing technique. The intent of this analysis was to determine if the smoothing technique 
worked consistently on all track types with either an increasing or decreasing effect of smoothing with window size or angle size (i.e., the interaction term is not significant). The interaction term was significant (GLM, $\mathrm{p}<0.001)$ for both the moving window regression and the censored angle smoothing techniques (Figure 4) but was not significant $(\mathrm{GLM}, \mathrm{p}=0.853)$ for the moving window average (Figure $5)$.
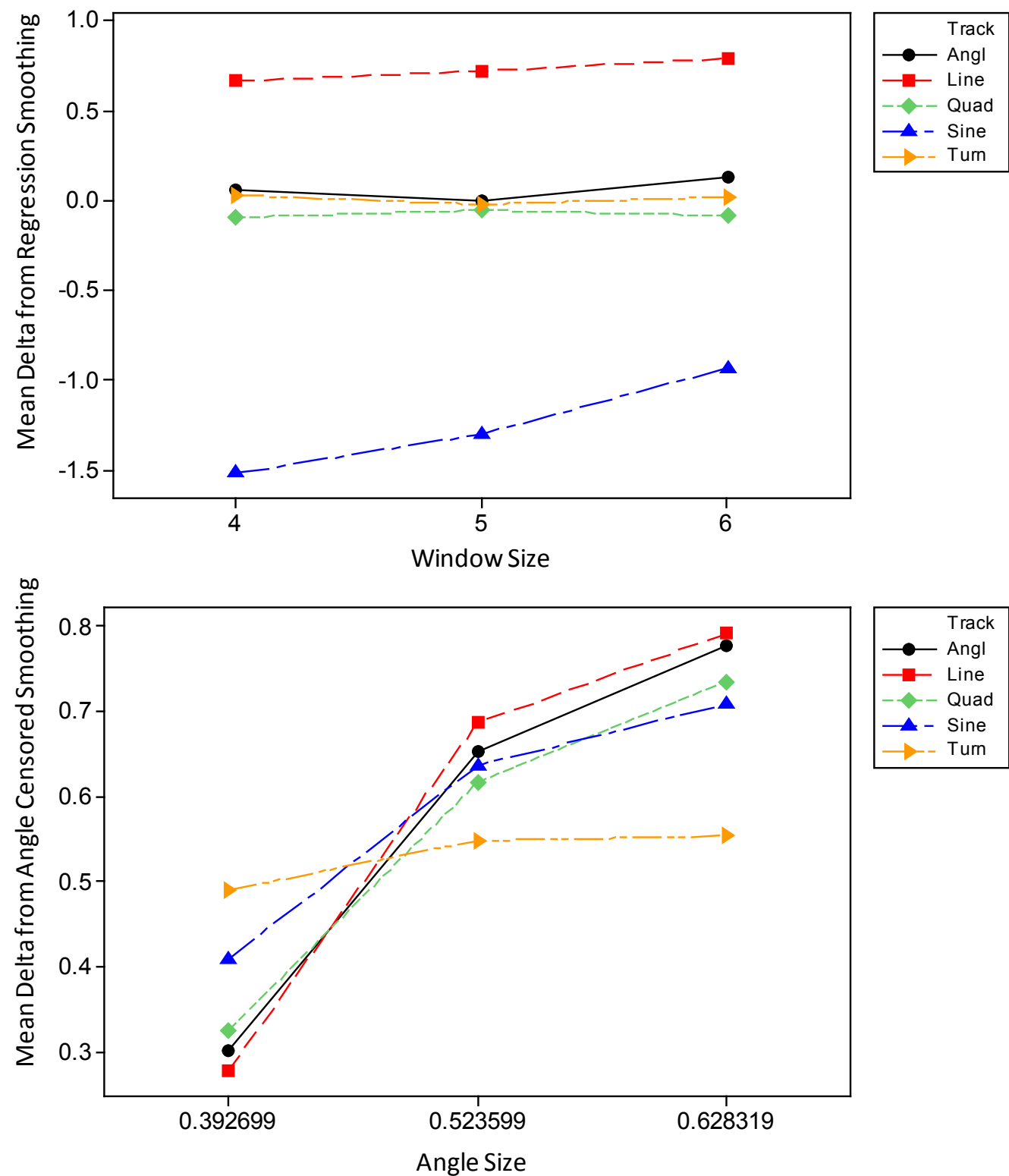

Figure 4. Interaction plots for the delta statistic for the IQR of the change in direction using regression smoothing (top) and angle censored smoothing (bottom). 


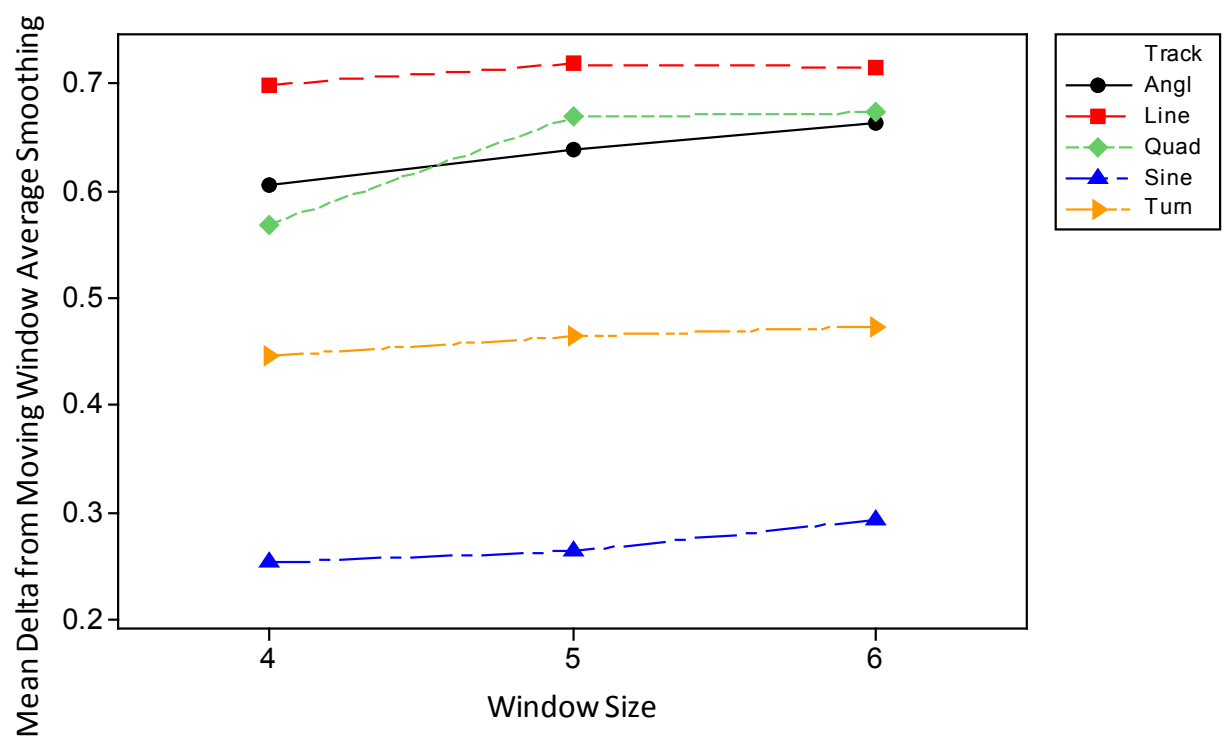

Figure 5. Interaction plots for the delta statistic for the IQR of the change in direction using moving window average smoothing.

The moving window average smoothing had the least effect on the sine wave (Sine) track and the greatest effect on the linear (Line) track (Figure 5). The smoothing effect on the angled (Angl) and quadratic (Quad) tracks was not significantly different (Tukey's Multiple Comparison test, $\mathrm{p}>0.05$ ), but the smoothing effect on all other pair-wise tracks were significantly different (Tukey's Multiple Comparison test, $\mathrm{p}<0.05$ ). The effect of smoothing with a window size of 6 time points was significantly greater, although only minimally, than a window size of 4 (Tukey's Multiple Comparison test, $\mathrm{p}<0.05$ ). Regression smoothing increased the IQR for the sine wave track (negative delta, Figure 4) and had a minimal effect on the angled, quadratic, and turnaround (Turn) tracks. The angle size chosen for the angle censored smoothing had a large increasing effect on all tracks except the turnaround track. Thus, moving window average smoothing with a window size of 6 time points was chosen as the preferred smoothing technique.

\subsection{Track Classification}

As previously stated, attributes of the tracks extracted from the video will provide information to classify tracks according to their identity. Track attributes that are currently extracted from video include measures of intensity, object size, rate of travel across the camera view, and track sinuosity. Thermal intensity is a function of the temperature radiated from an object and the contrast of the object with the background. The FieldPro 5x Thermal Imager measures thermal intensity in a relative manner rather than as an absolute temperature. This results in thermal values that are dependent upon ambient conditions. Differences in thermal values within video were used to define objects and tracks.

We did not attempt to identify an object that made the track based on its thermal signature because our hardware/software configuration would not enable us to do so. Although size is a key factor often used by human observers to identify different types and species of birds and bats, size on the video is also a function of distance from the camera. Because the camera view offers only a two-dimensional view of 
what was observed, the distance to the object is unknown such that the absolute size of the recorded object is unknown. At this time, object size is not reliable for identification because of unknown range to the object. Although different bird species may also be discernible by flight speed, use of distance traveled through the camera view per frame can be problematic in a two-dimensional realm. The distance an object travels per frame within the video is a function of its distance from the camera lens. Actual flight speed is influenced by wind speed and direction relative to the object's flight path at the time the object is recorded, as a bird flying into the wind would be traveling slower than the same bird traveling with the wind. Perceived flight speed is also a function of the aspect of flight relative to the plane of the camera lens. A bird flying directly at or away from the camera lens appears stationary, even though it may be moving at a relatively high rate of speed. Without range information to allow for threedimensional tracking, distance traveled per video frame is not a reliable indicator of actual flight speed.

Sinuosity, defined as the amount of direction change within the entire recorded flight track, is a characteristic that could be used to begin to classify recorded objects. The descriptive statistics of the change in direction are intended to separate the tracks into broad categories of potential bird and bat flight paths. Generally speaking, birds tend to fly straight (linear, quadratic, and angled) and bats tend to fly in a more erratic pattern (sine wave and turnaround). Larger birds tend to change direction less often or less abruptly than smaller birds or bats. Using the smoothed sinuosity data, forward-stepping discriminant analysis on standardized variables was used to determine the set of statistics that best separates these tracks. Cluster analysis with complete linkage and Euclidean distance was also used to assess the similarity between tracks. The correlation matrix of the pair-wise descriptive statistics of the change in direction using the average smoothed tracks with moving window size 6 smoothing was calculated (Table 2). The first and third quartiles (Q1 and Q3) were highly correlated $(|r| \geq 0.9)$ with the IQR, the range was highly correlated with the minimum and maximum, the skewness was highly correlated with kurtosis, and the standard deviation was highly correlated with the minimum, maximum, and the range. Thus, the Q1, Q3, range, skew, and standard deviation of the change in direction were not used in the multivariate cluster and discriminant analyses.

Table 2. Correlation matrix of the descriptive statistics of the change in direction using the moving window $($ size $=6)$ smoothing $(\mathrm{n}=27)$.

\begin{tabular}{|l|c|c|c|c|c|c|c|c|c|c|c|c|}
\hline Statistic & $\begin{array}{c}\text { Central } \\
\text { Skew }\end{array}$ & IQD & Kurtosis & Max & Mean & Median & Min & $\begin{array}{c}\text { No. Sign } \\
\text { Changes }\end{array}$ & Q1 & Q3 & Range & Skew \\
\hline IQD & -0.72 & & & & & & & & & & & \\
\hline Kurtosis & 0.19 & -0.15 & & & & & & & & & & \\
\hline Max & -0.59 & 0.84 & 0.33 & & & & & & & & & \\
\hline Mean & 0.47 & -0.63 & 0.13 & -0.51 & & & & & & & & \\
\hline Median & -0.55 & 0.11 & -0.07 & 0.11 & 0.48 & & & & & & & \\
\hline Min & 0.27 & -0.46 & -0.79 & -0.82 & 0.29 & 0.01 & & & & & & \\
\hline $\begin{array}{l}\text { \# Sign } \\
\text { Changes }\end{array}$ & -0.55 & 0.79 & 0.31 & 0.88 & -0.70 & -0.12 & -0.76 & & & & & \\
\hline Q1 & 0.72 & -0.96 & 0.16 & -0.79 & 0.77 & 0.01 & 0.43 & -0.80 & & & & \\
\hline
\end{tabular}


Table 2. (contd)

\begin{tabular}{|l|c|c|c|c|c|c|c|c|c|c|c|c|}
\hline Statistic & $\begin{array}{c}\text { Central } \\
\text { Skew }\end{array}$ & IQD & Kurtosis & Max & Mean & Median & Min & $\begin{array}{c}\text { No. Sign } \\
\text { Changes }\end{array}$ & Q1 & Q3 & Range & Skew \\
\hline Q3 & -0.65 & 0.95 & -0.13 & 0.83 & -0.43 & 0.25 & -0.45 & 0.69 & -0.83 & & & \\
\hline Range & -0.39 & 0.61 & 0.66 & 0.92 & -0.38 & 0.03 & -0.98 & 0.83 & -0.58 & 0.60 & & \\
\hline Skew & -0.16 & 0.16 & -0.98 & -0.30 & -0.12 & 0.05 & 0.78 & -0.30 & -0.17 & 0.13 & -0.64 & \\
\hline $\begin{array}{l}\text { Standard } \\
\text { Deviation }\end{array}$ & -0.53 & 0.78 & 0.46 & 0.98 & -0.50 & 0.06 & -0.90 & 0.89 & -0.74 & 0.76 & 0.97 & -0.45 \\
\hline
\end{tabular}

Cluster analysis using the eight descriptive statistics suggests that the turnaround and sine wave tracks are different from the linear, quadratic, and angled tracks (Figure 6). The centroids of the two sine wave track groups (blue and pink lines) were no more than $26 \%$ similar to the other tracks (Table 3 ). The turnaround track (orange) was more similar to the angled and quadratic tracks than the sine wave tracks. The linear, quadratic, and angled tracks were on average greater than $50 \%$ similar.

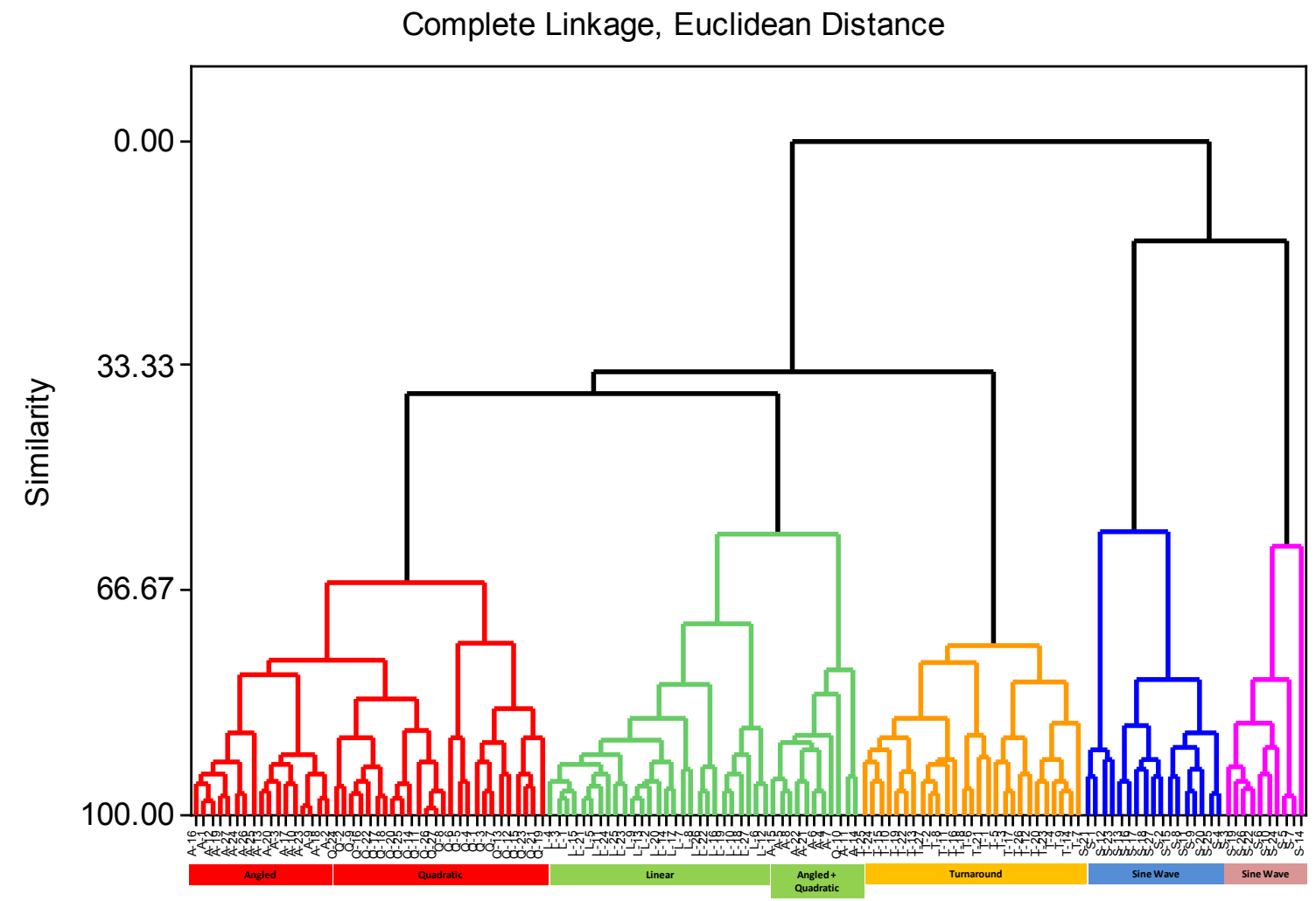

Figure 6. Cluster analysis of the 135 tracks. Tracks that are at least $50 \%$ similar are connected with a similar line color.

Discriminant analysis on the standardized variables used in the cluster analysis was able to correctly classify $100 \%$ of the tracks into the five track categories (linear, quadratic, angled, sine wave, and turnaround). Only two variables were dropped from the model (central skew and minimum change in direction). Two eigenvalues were able to explain $91 \%$ of the variability (Figure 7). The kurtosis had the 
greatest discriminant power followed by the mean change in direction as can be seen by the magnitude of the coefficients of the canonical variables (Table 4).

Table 3. Similarity matrix between cluster centroids.

\begin{tabular}{|l|c|c|c|c|c|c|}
\hline Color & $\begin{array}{c}\text { Number of } \\
\text { Tracks in } \\
\text { Cluster }\end{array}$ & Red & Green & Orange & Blue & Pink \\
\hline Red & 43 & $100 \%$ & $56 \%$ & $32 \%$ & $4 \%$ & $21 \%$ \\
\hline Green & 38 & $56 \%$ & $100 \%$ & $28 \%$ & $0 \%$ & $3 \%$ \\
\hline Orange & 27 & $32 \%$ & $28 \%$ & $100 \%$ & $11 \%$ & $26 \%$ \\
\hline Blue & 17 & $4 \%$ & $0 \%$ & $11 \%$ & $100 \%$ & $55 \%$ \\
\hline Pink & 10 & $21 \%$ & $3 \%$ & $26 \%$ & $55 \%$ & $100 \%$ \\
\hline
\end{tabular}

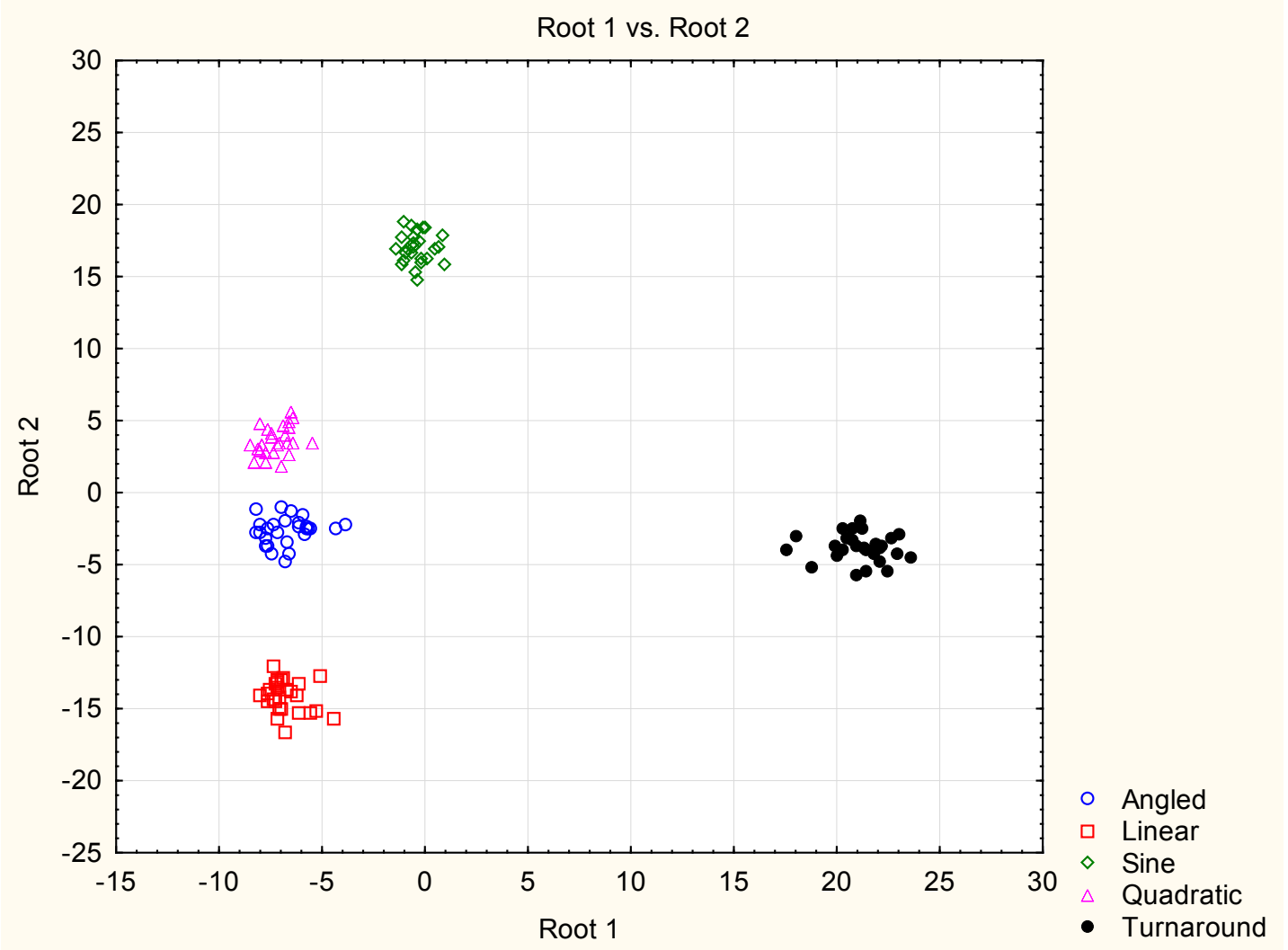

Figure 7. Discriminant analysis of the 135 tracks. 
Table 4. Coefficients for the canonical variables.

\begin{tabular}{|l|c|c|}
\hline \multicolumn{1}{|c|}{$\begin{array}{c}\text { Standardized Descriptive Statistic } \\
\text { for the Change in Direction }\end{array}$} & Root 1 & Root 2 \\
\hline Kurtosis & 10.55 & -0.9643 \\
\hline Mean & 0.56 & -7.6155 \\
\hline Max & 0.20 & 2.5520 \\
\hline IQR & 0.90 & 0.7932 \\
\hline Number sign changes & 0.89 & 0.6124 \\
\hline Median & -0.05 & 0.1724 \\
\hline Constant & 0.00 & 0.00 \\
\hline Eigenvalue & 122 & 109 \\
\hline Cumulative Percentage of Variability Explained & $48 \%$ & $91 \%$ \\
\hline
\end{tabular}

A new flight path was created from random sections of each of the five original flight paths to determine how the discriminant function analysis would classify a track with features of each representative track type (Figure 8 ). The $\mathrm{X}-\mathrm{Y}$ data were smoothed using a moving window average with a window size of six time points. The direction and change in direction between each successive time point was calculated as described above. The descriptive statistics on the change in direction used in the discriminant function (kurtosis, mean, max, IQR, number of sign changes, and the median) were calculated and standardized based on the mean and standard deviation of the original data set $(\mathrm{n}=135$, Table 5). The discriminant scores (Root 1 and Root 2) were calculated based on the coefficients presented in Table 4. Finally, the Euclidean distance to each centroid (Table 6) and the probability of membership to each of the track categories was calculated (Table 7). As expected, the new track made up of portions of all theoretical flight path types was not closely related to any single theoretical flight path (Figure 9). The complexity of this new flight path resembled what we expected would most represent the erratic flight of a bat. In general, flight paths with characteristics similar to sine waves and the turnaround paths are hypothesized to be representative of a bat, and the location of the new track plotted in canonical space within space supported our theory. 


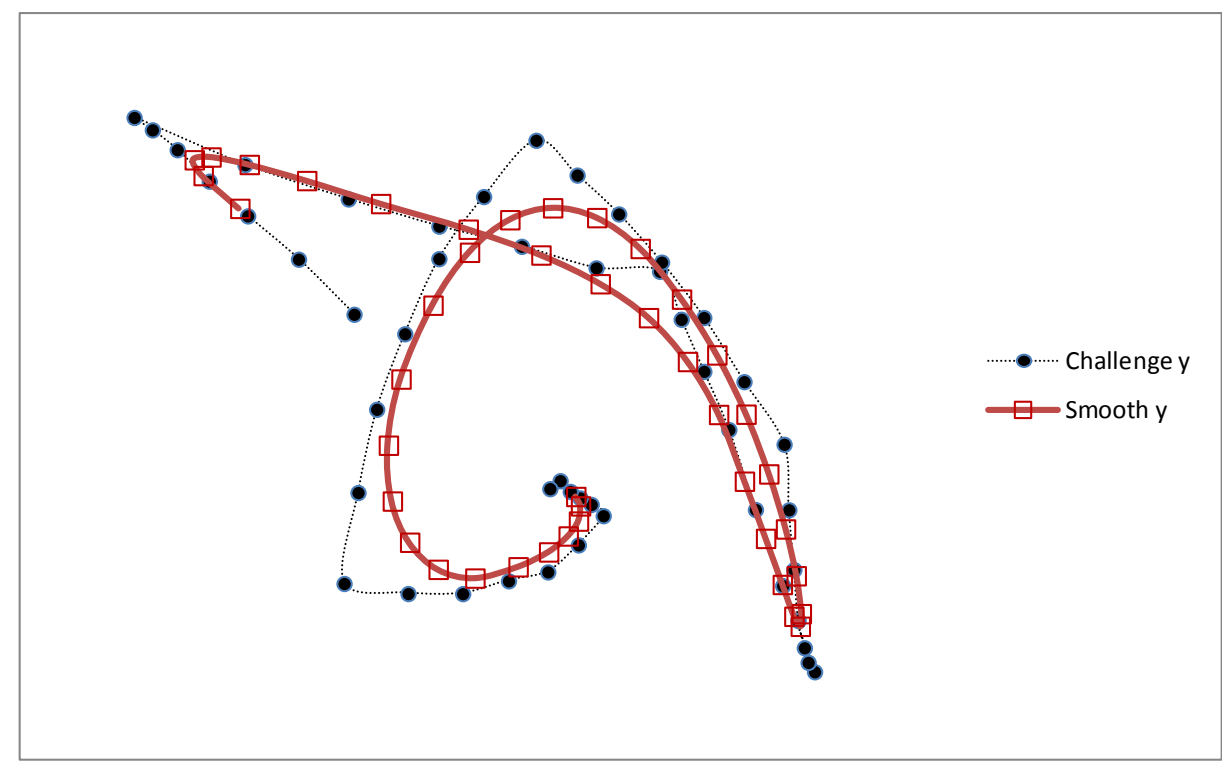

Figure 8. A 2-dimensional representation of the new flight path used to challenge the discriminant function classification process.

Table 5. Mean and standard deviation for standardizing the descriptive statistics on the change in direction used in the discriminant function.

\begin{tabular}{|c|c|c|c|c|c|c|}
\hline Statistic & IQR & Kurtosis & Maximum & Mean & Median & $\begin{array}{c}\text { Number of } \\
\text { Sign } \\
\text { Changes }^{(a)}\end{array}$ \\
\hline Mean & 0.16 & 5.10 & 0.40 & -0.03 & -0.02 & 2.93 \\
\hline Stdev & 0.12 & 8.95 & 0.35 & 0.02 & 0.02 & 2.55 \\
\hline
\end{tabular}

(a) This value is dependent on the length of the track. Therefore, a new observation must be calculated as a proportion of the total number of $C_{i}$ consecutive pairs and then multiplied by $\mathrm{n}=$ 30 (the number in the modeled tracks).

Table 6. Means (centroid) of the canonical variables (Root 1 and Root 2) for each track.

\begin{tabular}{|l|c|c|}
\hline \multicolumn{1}{|c|}{ Track } & Root 1 & Root 2 \\
\hline Linear & -6.76 & -14.11 \\
\hline Quadratic & -7.26 & 3.54 \\
\hline Angled & -6.69 & -2.64 \\
\hline Sine & -0.40 & 17.02 \\
\hline Turnaround & 21.11 & -3.82 \\
\hline
\end{tabular}


Table 7. Distance from each centroid and probability of membership to each track category for the challenge track.

\begin{tabular}{|l|c|c|}
\hline \multicolumn{1}{|c|}{ Track } & Distance from Centroid & Probability \\
\hline Linear & 20.4 & 0.75 \\
\hline Quadratic & 14.0 & 0.83 \\
\hline Angled & 13.8 & 0.83 \\
\hline Sine wave & 17.2 & 0.79 \\
\hline Turnaround & 15.4 & 0.81 \\
\hline
\end{tabular}

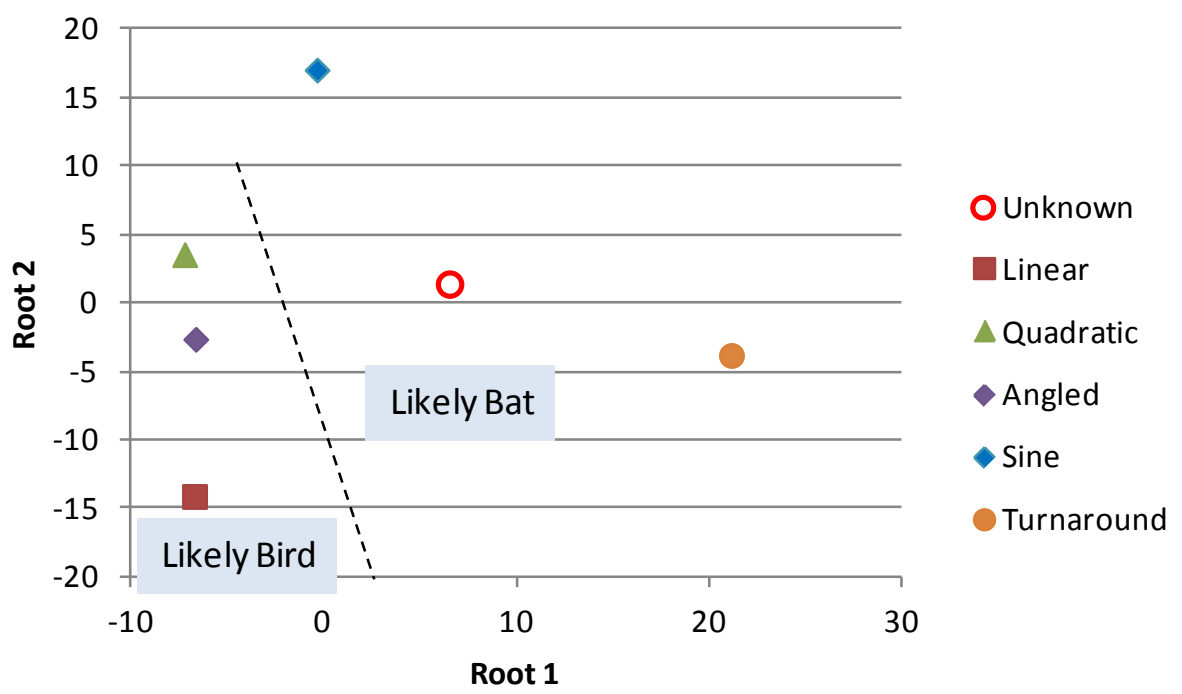

Figure 9. Location in canonical space of the unknown challenge track.

These analyses indicate that track sinuosity could be used as a factor to begin to classify tracks into broad functional groups, although the degree to which sinuosity could be used has not been determined. Data on flight path characteristics is not currently available. Our theory that sinuosity could be used to classify paths is based on first-hand observations and represents anecdotal information at this time.

\subsection{Successes, Challenges and Future Development}

We were able to develop methods to automatically identify targets recorded with infrared video, including performing Video Peak Store methods with computer code. We also developed methods to extract information describing flight tracks of the identified objects. Our confirmatory analyses to determine the effect of random variation on flight track sinuosity enabled the development and testing of data smoothing techniques to distinguish tracks based on sinuosity. Our video library will allow us to describe and classify bird groups and species we observed in marine environments. However, automated identification, enumeration, and classification of tracks are complicated by a number of factors. Tracks crossing in space and time are sometimes not discernible as separate tracks. Because parameters within 
our VPS process were based on the original sample video, a 10-second window was selected as the amount of time needed to contain entire tracks. If many objects are rapidly moving through the view in the same direction, tracks become superimposed within the 10-second window and may not be discernible with the current algorithm configuration. This case was observed when many gulls were flying to a particular roost near the camera, and a strong crosswind resulted in many birds flying at the same elevation in rapid succession. The case also could occur when flocking birds such as shorebirds or migrant passerines pass through the camera view. Current thought would be to either create an automated window-length setting based on analyses of the actual video recorded by the user and/or allow the user to manually define the length of time based on video post-processing results. These capabilities do not currently exist within our algorithm development.

Our current VPS process also results in each 10-second window overlapping the previous and successive windows by 5 seconds. This methodology ensures every track would be fully contained within at least one 10-second window. However, it also results in partial tracks being labeled as new tracks in adjacent time windows which then results in an overestimate of the number of observed tracks. However, attributes of partial tracks can be used to identify them as such for removal from the dataset. We are currently developing automated methods to extract duplicate and partial tracks identified during the VPS process.

A key component for classification of phenomena recorded with an infrared video camera is a description of phenomena expected to be encountered. This description must be in quantifiable terms that are extractable from video. In order to begin to distinguish among various phenomena that produced tracks within our thermal video, measures of intensity, object size, relative speed, and direction change are calculated for each track. However, without range information, measures of size and speed are of limited use. Direction change shows promise as a variable that could be used to classify tracks into broad categories. Another flight characteristic often used by human observers to identify birds or bats is wingbeat frequency. Wing beats are often discernible within the sample video as well as our video library, as current thermal imaging technologies provide sufficient resolution to enable viewing of wing beats of most seabirds at ranges that would be of interest with respect to impacts from offshore wind turbines. However, the likelihood that any given track can be accurately classified based on a combination of flight characteristics such as sinuosity and wing-beat frequency, neither of which is necessarily influenced by range, depends on the availability of information that mathematically describes these flight characteristics of known or expected targets. Previous studies, including development of the Thermal Animal Detection System (Desholm 2003), recorded numerous bird species while flying through a thermal imaging video camera view. Betke et al. (2008) used thermal imaging to count bats emerging from a colony. Although the level of annotation that accompanies such video sources is unknown, Desholm (2003) published still images of known bird species taken from thermal video. Our acquisition of an annotated video library was conducted in part to enable us to begin to describe phenomena with a known identity using any or all information that could be obtained from infrared video. Our efforts will not be sufficient to describe all phenomena that could be recorded at other times or locations. Instead, these efforts will begin the characterization process and allow the subsequent use of software technologies to detect and classify objects that could be at risk from offshore wind energy 


\subsection{References}

Betke M, DE Hirsh, NC Makris, GF McCracken, M Procopio, NI Hristov, S Tang, A Bagchi, JD Reichard, JW Horn, S Crampton, CJ Cleveland, and TH Kunz. 2008. "Thermal imaging reveals significantly smaller Brazillian free-tailed bat colonies than previously estimated." Journal of Mammalogy 89(1):18-24.

d'Entremont M. 2010. "How does the accuracy of data on avian movement vary with radar methodology?" In Wind Wildlife Research Meeting VIII, pp. 122-126. National Wind Coordinating Collaborative, Lakewood, Colorado.

Davenport J. 2010. "Challenges and solutions for using radar at offshore wind energy developments." In Wind Wildlife Research Meeting VIII, pp. 135-138. National Wind Coordinating Collaborative, Lakewood, Colorado.

Desholm M. 2003. Thermal Animal Detection System (TADS): Development of a Method for Estimating Collision Frequency of Migrating Birds at Offshore Wind Turbines. NERI Technical Report 440, National Environmental Research Institute, Ministry of the Environment, Denmark. Available from http://www.dmu.dk/udgivelser/faglige+rapporter/ (September 2012).

Gauthreaux SA Jr. and JW Livingston. 2006. "Monitoring bird migration with a fixed-beam radar and a thermal-imaging camera." Journal of Field Ornithology 77(3):319-328.

Johnson G. 2010. "Relationships between bat fatality and weather, marine radar, AnaBat, and night vision data at a wind energy facility in the Midwest." In Wind Wildlife Research Meeting VIII, pp. 21-24. National Wind Coordinating Collaborative, Lakewood, Colorado.

Svedlow A. 2011. "Offshore surveys for bird and bats-Block Island wind farm." Presented to the EnergyOcean International Conference, July 15, 2011, Portland, Maine. Tetra Tech, Inc., Portland, Maine. Available from http://joomla.wildlife.org/Maine/images/WindEnergy/

4 asvedlow\%20tetra\%20tech\%20metws\%20may\%202011.pdf (September 2012).

Zehnder S, S Åkesson, F Liechti, and B Bruderer. 2001. "Nocturnal autumn bird migration at Falsterbo, South Sweden.” Journal of Avian Biology 32:239-248. 



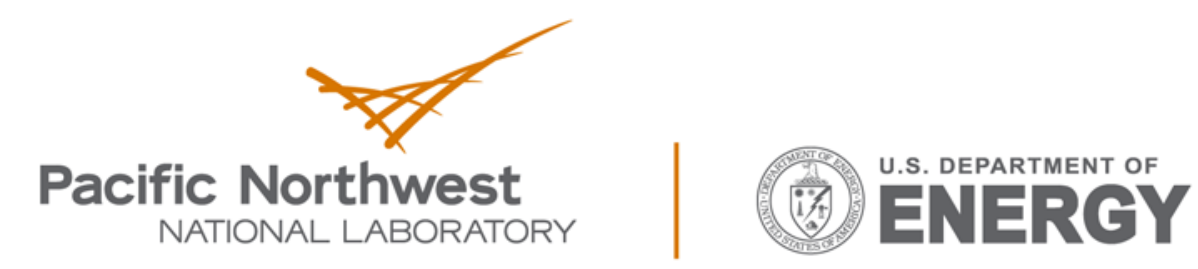

Proudly Operated by Battelle Since 1965

902 Battelle Boulevard

P.O. Box 999

Richland, WA 99352

1-888-375-PNNL (7665)

www.pnnl.gov 